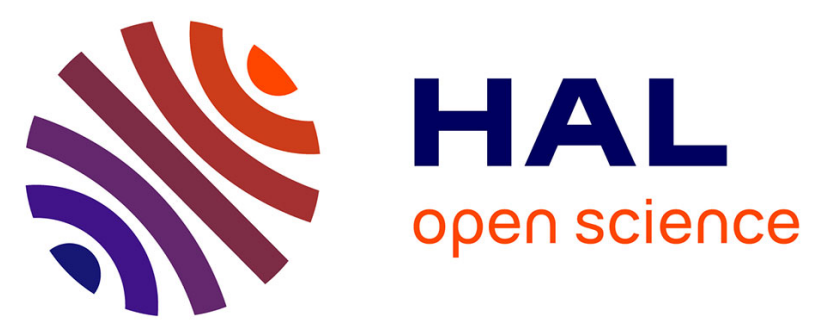

\title{
Preparation of Substituted 2 H -Pyrans via a Cascade Reaction from Methyl Coumalate and Activated Methylene Nucleophiles
}

Liang Chang, Kristína Plevová, Serge Thorimbert, Luc Dechoux

\section{- To cite this version:}

Liang Chang, Kristína Plevová, Serge Thorimbert, Luc Dechoux. Preparation of Substituted 2 H -Pyrans via a Cascade Reaction from Methyl Coumalate and Activated Methylene Nucleophiles. Journal of Organic Chemistry, 2017, 82 (10), pp.5499-5505. 10.1021/acs.joc.7b00761 • hal-01520277

\section{HAL Id: hal-01520277 \\ https: / hal.sorbonne-universite.fr/hal-01520277}

Submitted on 10 May 2017

HAL is a multi-disciplinary open access archive for the deposit and dissemination of scientific research documents, whether they are published or not. The documents may come from teaching and research institutions in France or abroad, or from public or private research centers.
L'archive ouverte pluridisciplinaire HAL, est destinée au dépôt et à la diffusion de documents scientifiques de niveau recherche, publiés ou non, émanant des établissements d'enseignement et de recherche français ou étrangers, des laboratoires publics ou privés. 
The $2 H$-pyran core is widely encountered in natural products ${ }^{1}$ and is often used as a key intermediate in total synthesis of natural products. ${ }^{2}$ Since $2 H$-pyrans are in equilibrium with 1 oxatrienes by a $6 \pi$ electrocyclic process, 1 -oxatrienes generally afford an entry into the $2 \mathrm{H}$-pyran skeleton (Figure 1). The $6 \pi$ disrotatory process has been already discussed at different levels of theory. ${ }^{3}$ The equilibrium between $2 \mathrm{H}$-pyrans $\mathbf{A}$ and 1oxatrienes $\mathbf{B}$ depends on many factors such as the presence of electron-withdrawing substituents or increased steric interactions. The most common entry into the 1-oxatriene core is the Knoevenagel reaction (most generally condensation between an enal and a cyclic 1,3-dicarbonyl compound). From a synthetic point of view, the tandem Knoevenagel / $6 \pi$ electrocyclization (Figure 1) can be considered as a formal $[3+3]$ cycloaddition. ${ }^{4,5}$ Conjugated 1-oxatrienes can also be obtained through a Claisen rearrangement of propargyl vinyl ethers ${ }^{6}$ or cycloisomerization of diynols. ${ }^{7}$ Other strategies towards $2 H$-pyrans that do not involve the " 1 -oxatriene pathway" but rather rely on formal [4+2]- or [3+3]-cycloaddition have been reported. ${ }^{8,9}$

We present a study enabling a general synthesis of tetrasubstituted $2 \mathrm{H}$-pyrans by addition of methylene active nucleophiles D to methyl coumalate (MC). We have recently demonstrated that Grignard reagents add regioselectively on the $\mathrm{C} 6$ of $\mathbf{M C} .^{10}$ Our hypothesis was that MC should react with the same regiochemistry with stabilized nucleophiles D like keto-esters or diketones in a tandem 1,6-Michael addition - $6 \pi$-electrocyclic ring opening $-1,5 \mathrm{H}$ shift reaction offering an entry to 1oxatrienes B'. This highly strained 1-oxatriene bearing two electron-withdrawing groups should through a final $6 \pi$ electrocyclization, deliver the $2 H$-pyran systems A' (Figure 1).
Figure 1. Approaches to 2H-Pyrans

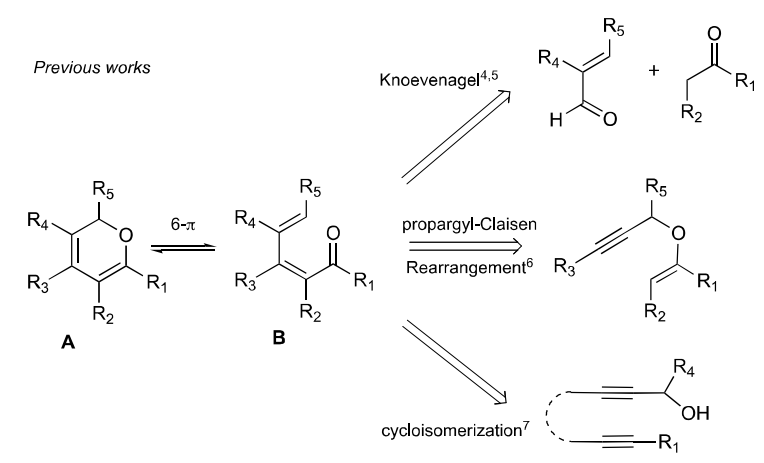

This work

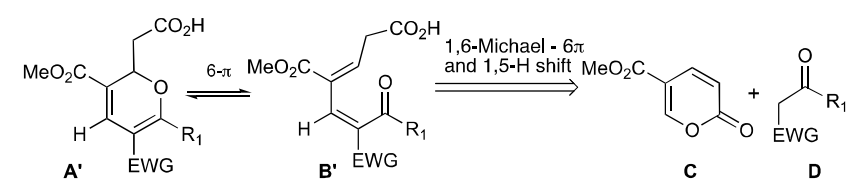

This hypothesis was also based on a report of the literature ${ }^{11}$ in which the aceto-phenylsulfone reacted with $\mathbf{M C}$ at high temperature to give the corresponding decarboxylated $2 \mathrm{H}$-pyran in $67 \%$ yield. Only one example was described and we decided to reinvestigate the reaction conditions and to study the scope and limitations. We demonstrate here that careful control of the temperature is crucial and, in addition to the previously reported $2 H$-pyran, that 2-(2H-pyran-2-yl)acetic acids are also available in good yields. We also proposed a modified mechanism that takes into account some of our previous results. 
We started our investigation with methyl acetoacetate (MAA) as a model nucleophile in the cascade reaction (Table 1 ). The first attempt was carried out with $20 \mathrm{~mol} \%$ of cesium carbonate in DCM at room temperature for $15 \mathrm{~h}$. The expected $2 H$-pyran 1a was obtained in low yield due to low conversion (MC recovered in $75 \mathrm{~mol} \%$, see SI, Table S1). A stoichiometric amount of cesium carbonate gave compound 1a with moderate yield (60\%) (Table 1, entry 1$)$.

Table 1. Optimization of the Reaction Conditions ${ }^{\mathrm{a}}$

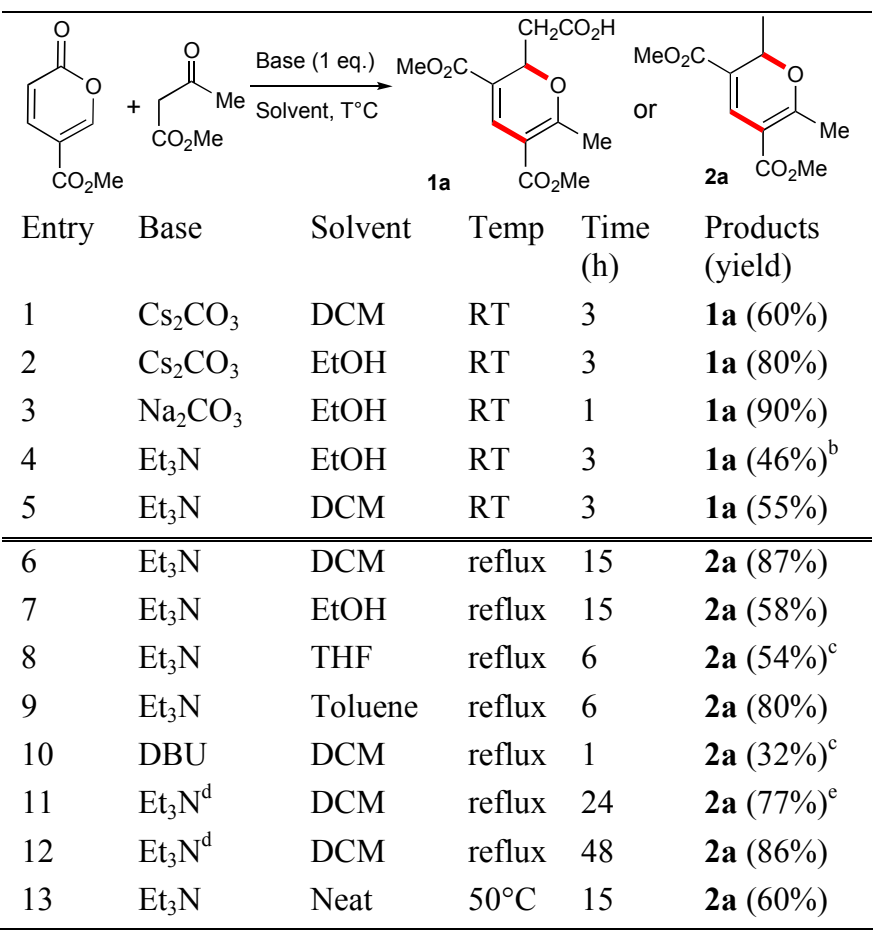

${ }^{a}$ Reaction conditions: All reactions were performed with $\mathrm{MC}$ ( $1 \mathrm{mmol})$ and MAA $(1 \mathrm{mmol})$, in $5 \mathrm{~mL}$ of organic solvent. ${ }^{\mathrm{b}} 54 \%$ of $\mathrm{MC}$ was observed in the crude reaction mixture, determined by ${ }^{1} \mathrm{H}$ NMR. ${ }^{\mathrm{c}}$ Degradation was observed. ${ }^{\mathrm{d}} 20 \mathrm{~mol} \%$ of $^{\mathrm{Et}} \mathrm{t}_{3} \mathrm{~N}$. ${ }^{\mathrm{e}} 18 \%$ of $\mathrm{MC}$ was observed in the crude reaction mixture, determined by ${ }^{1} \mathrm{H}$ NMR

$2 H$-Pyran 1a was obtained with better yield when the reaction was performed in EtOH with either cesium or sodium carbonate (entries 2-3). At $\mathrm{rt} \mathrm{NEt}_{3}$ gave moderate conversions and yields using either EtOH or DCM as solvents (entries 4-5). Interestingly, after prolonged reaction time, some traces of the decarboxylated $2 H$-pyran 2a were observed (see SI, Table S1). In order to favour the complete decarboxylation, the reaction mixture was heated to reflux for $15 \mathrm{~h}$ in DCM. Product $\mathbf{2 a}$ was thus isolated in good yield ( $87 \%$, entry 6$)$. Changing DCM for toluene, EtOH or THF did not improve the yield of $\mathbf{2 a}$ (entries 7-9) and the use of DBU instead of $\mathrm{NEt}_{3}$ in DCM gave only modest results (entry 10). Interestingly, the reaction was successfully tested with a catalytic amount of $\mathrm{Et}_{3} \mathrm{~N}$. Complete conversion of MC into the expected decarboxylated $2 \mathrm{H}$-pyran 2a was reached in $48 \mathrm{~h}$ with $86 \%$ yield (entry 12 , see also SI, Table S1 for shorter reaction times). A solvent-free reaction led to the formation of the expected compound $\mathbf{2 a}$, however in a moderate $60 \%$ yield after 15 hours at $50^{\circ} \mathrm{C}$ (entry 13 ).

Having in hand the best conditions to synthesize either $2 \mathrm{H}$ pyran 1a (entry 3) or its corresponding decarboxylated analogue 2a (entry 6), we explored the scope and limitations of these reactions.
The data in Table 2 illustrate the reaction with $\mathrm{Na}_{2} \mathrm{CO}_{3}$ in $\mathrm{EtOH}$ for $1 \mathrm{~h}$ at room temperature. 1,3-Ketoesters gave the expected $2 H$-pyrans 1a-c,e,f in yields ranging from $86 \%$ to $92 \%$. Diverse functions such as methylether (1c), halogen (1e) or a second ester function (1f) gave the expected products whereas 1,3-diketones gave moderate to good yields. For example, the symmetrical 1,3-pentanedione gave the $2 H$-pyran $1 \mathrm{~g}$ in $87 \%$ yield whereas the non-symmetrical acetoacetophenone gave the $2 H$-pyrans $\mathbf{1 h}$ and $\mathbf{1 h}$ ' in a $3 / 1$ mixture, which could not be purified by flash chromatography, reflecting the two diastereoisomeric 1-oxatriene intermediates $5 \mathbf{h}$ $(\mathrm{EWG}=\mathrm{COPh} \mathrm{R}=\mathrm{Me})$ and $\mathbf{5 h}$ ' $(\mathrm{EWG}=\mathrm{COMe}$ and $\mathrm{R}=\mathrm{Ph})($ see Scheme 1).

The purifications of some of $2 H$-pyrans 1 were not satisfying. We surmised that direct esterification of the carboxylic acid function following Steglich procedure ${ }^{12}$ could solve the problem. Compounds $\mathbf{3 d} \mathbf{d}, \mathbf{3 i}, \mathbf{3 j}$ were efficiently prepared in a onepot manner with yields from $52 \%$ to $72 \%$ (Table 2 ).

Table 2. Substrate Scope of the Tandem Michael / $6 \pi$ / $1,5 \mathrm{H}$ shift / $6 \pi$ Reaction at Room Temperature ${ }^{\mathrm{a}}$

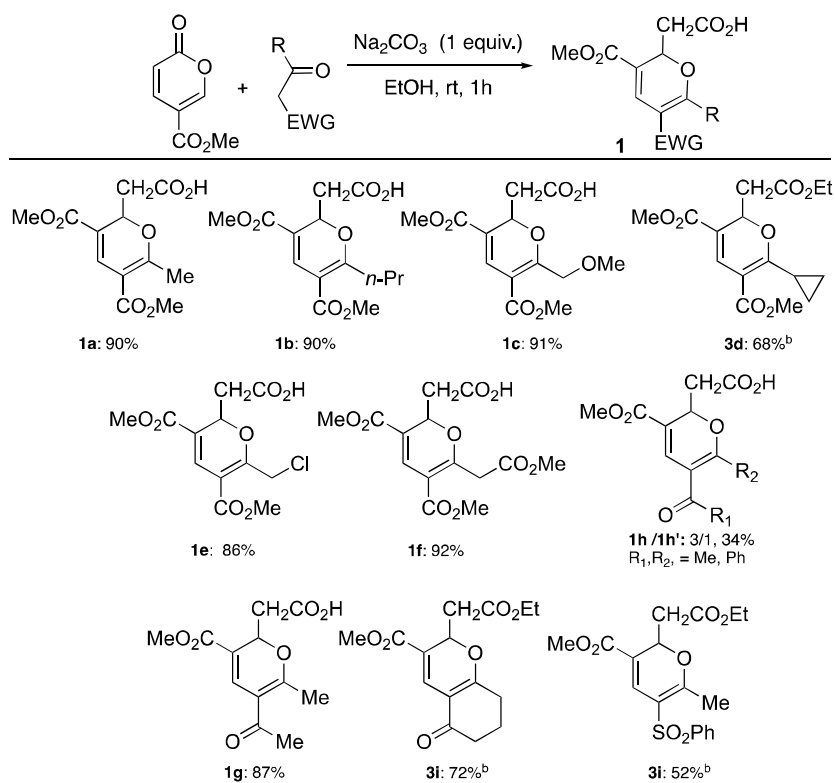

a Reaction conditions : MC/Methylene active nucleophile $/ \mathrm{Na}_{2} \mathrm{CO}_{3}(1 / 1 / 1)$ in EtOH for $1 \mathrm{~h} .{ }^{\mathrm{b}} 5 \mathrm{~mL}$ of DCM, 1 equiv. of EDC.HCl and $20 \mathrm{~mol} \%$ of DMAP were directly added to the reaction mixture after $1 \mathrm{~h}$ and stirred $15 \mathrm{~h}$.

We next examined the outcome of the cascade reactions in the presence of $\mathrm{NEt}_{3}$ in DCM under reflux. Under these conditions, more than 20 examples of $2 H$-pyrans 2 were synthesized. Results are reported in Table 3 . The decarboxylated $2 H$-pyrans 2a-f were prepared from the 1,3-ketoesters in excellent yields. Again, ether, halogen or ester moieties were tolerated in this cascade reaction. Satisfyingly and in contrast to the first conditions, the crowded $2 H$-pyran $\mathbf{2} \mathbf{k}$ and the aromatic $2 H$-pyrans

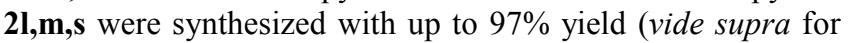
mechanistic explanations). The 4-hydroxycoumarin gave the expected tricyclic product $\mathbf{2 n}$ in $82 \%$ yield. In these conditions, $\beta$-oxosulfones led to $2 H$-pyrans $\mathbf{2} \mathbf{j}$,t in almost quantitative yield. $^{11}$ 
Table 3. Substrates Scope of the Decarboxylative Tandem Reaction $^{\text {a }}$
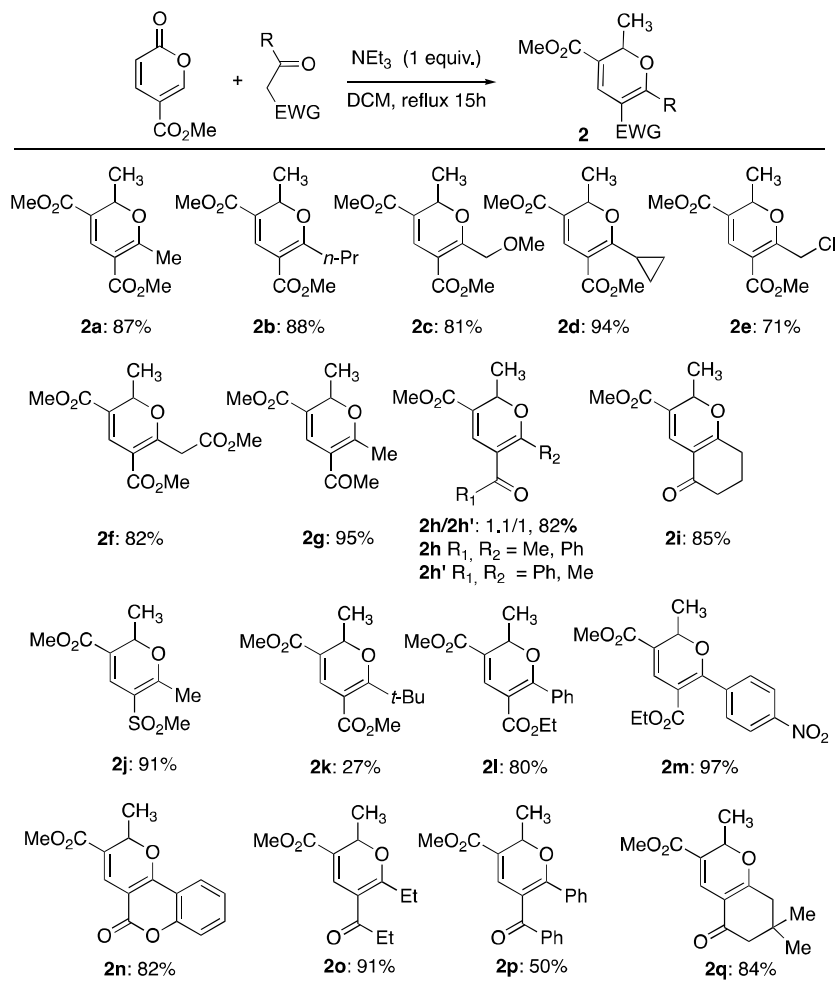

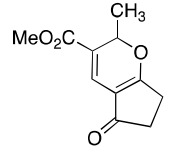

2r: $96 \%$
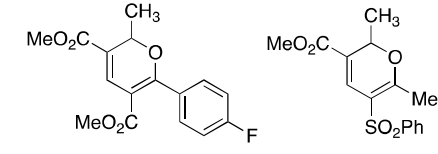

2s: $82 \%$
${ }^{a}$ Reaction conditions : MC/Methylene active nucleophile/ $\mathrm{Et}_{3} \mathrm{~N}$ $(1 / 1 / 1)$ in DCM reflux for $15 \mathrm{~h}$.

Various 1.3-diketones could also lead to the expected $2 \mathrm{H}$ Pyrans in moderate to excellent yields. Even aromatic diketones, which were unproductive at $\mathrm{rt}$ produced the expected pyrans $\mathbf{2 p}$ albeit in moderate yields. Again, acetoacetophenone gave a 1.1/1 mixture of isomers $\mathbf{2 h}$ and $\mathbf{2 h}$ ' in good yield $(82 \%)$. This result seems to indicate that there is no stereoselectivity in the proton shift step (see vide supra for mechanism). Finally $2 H$-pyrans $\mathbf{2} \mathbf{i}, \mathbf{q}, \mathbf{r}$ resulting from the addition of 5- and 6-membered cyclic diketones were isolated in good yields (84-96\%).

Our proposal for the mechanism of this cascade reaction might involve a 1,6-addition of the enolate on MC followed by a 6$\pi$-electrocyclic ring opening ${ }^{13}$ leading to the dienoic carboxylates 4 (Scheme 1) followed by a [1,5]-H shift through a deprotonation-reprotonation sequence. We ruled out a $[1,5]-\mathrm{H}$ sigmatropic shift due to high steric constraint in the required planar transition state due to the cis,cis configuration of the dienoic system $4 .{ }^{14} \mathrm{~A}$ further rapid $6 \pi$ electrocyclization would lead to the $2 H$-pyran 1' from oxatriene 5. This last electrocyclic reaction seemed to be a rapid process since oxatriene intermediate $\mathbf{5}$ was never detected. This observation suggests that even during the formation of $2 \mathrm{H}$-pyran $\mathbf{2}$, the mechanism involves intermediate 1'. This proposition is confirmed by the fact that $2 H$-pyran 1a, treated with $\mathrm{NEt}_{3}$ in DCM delivered, after $15 \mathrm{~h}$ at reflux, the corresponding decarboxylated $2 \mathrm{H}$-pyran 2a in $95 \%$. The mechanistic pathway to $2 H$-pyrans 2 implies that decarboxylation may operate on the transient 1-oxatriene 5 giving the stabilized enolate $\mathbf{6}$ which, after reprotonation from ammonium, gives the oxatriene 7 and regenerates $\mathrm{NEt}_{3}$ (Table 1 , entry 12). The mechanistic pathway ends with the rapid $6 \pi$ electrocyclization delivering the stable $2 \mathrm{H}$-pyran 2.

Scheme 1. Proposed Unified Mechanism for Cascade Reaction Leading to $2 H$-pyrans 1 and 2

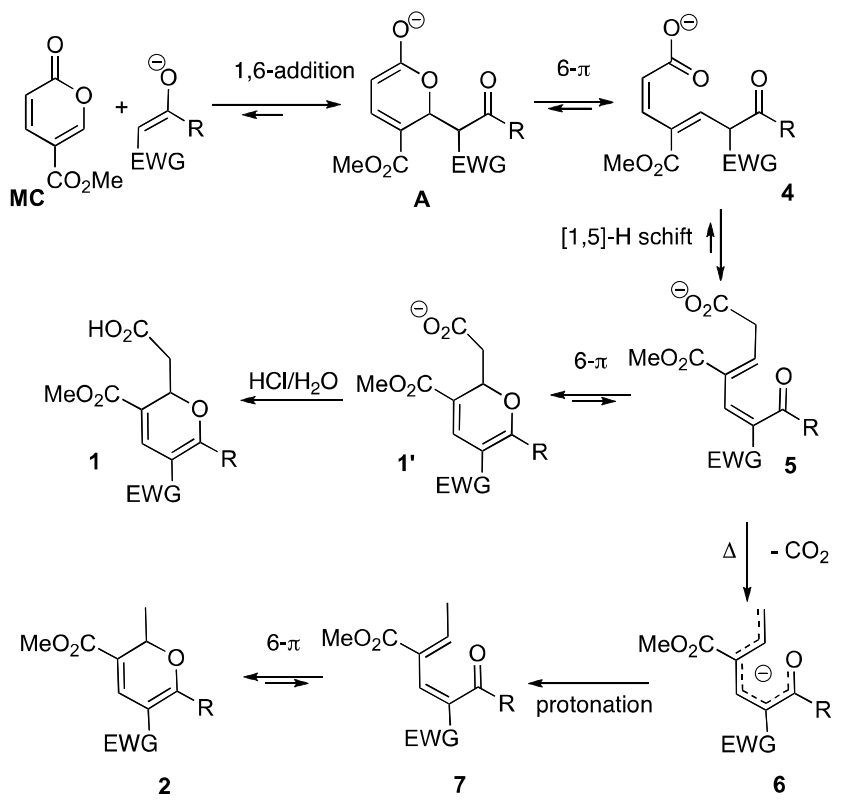

A possible rationale for the modest yields obtained with aromatic di-ketones at rt could be explained by steric factors. The planarity of the substituents of the double bonds $(\mathrm{EWG}=\mathrm{COAr}$ and $\mathrm{R}=\mathrm{Ar}$ ) in the $6 \pi$ transition state is required for the transformation of 1-oxatriene 5 to $2 H$-pyran 1'. Since the $6 \pi$ electrocyclization was inhibited and all the steps are reversible, starting materials were recovered accompanied by complex mixture of by-products. In contrast, by heating the reaction mixture, intermediate 5 was irreversibly converted to enolate $\mathbf{6}$ which after reprotonation and 6- $\pi$ electrocyclization gave the stable $2 \mathrm{H}$-pyran 2.

In summary, we described here a rapid and efficient synthesis of more than thirty stable tetrasubstituted $2 \mathrm{H}$-pyrans $\mathbf{1 , 2}$ and $\mathbf{3}$. The reactions to 1 or 2 proceed through a cascade 1,6-Michael / $6 \pi$ electrocyclic ring opening / [1,5]-H transfer / (decarboxylation) / $6 \pi$ electrocyclization reaction. In addition to the broad substrate scope, this methodology has the advantage of using readily available starting materials and mild conditions. The resulting $2 H$-pyrans bear functional groups, which should allow chemoselective transformations useful in total synthesis. ${ }^{2}$ Secoiridoid monoterpenes such as secologanin or gentiopicroside are also potential targets with biological activities of interest. ${ }^{15}$

\section{EXPERIMENTAL SECTION}

General. All reactions were carried out under argon atmosphere with magnetic stirring in dry and distillated solvents. ${ }^{1} \mathrm{H}$ and ${ }^{13} \mathrm{C}$ NMR spectra were recorded at $400 \mathrm{MHz}$ for ${ }^{1} \mathrm{H}$ nuclei, $100 \mathrm{MHz}$ for ${ }^{13} \mathrm{C}$ nuclei. Chemical shifts are reported in $\delta$ units, parts per million (ppm) using, for ${ }^{1} \mathrm{H}$ and ${ }^{13} \mathrm{C}$, solvent residual peak as internal standard references: chloroform $(7.26 \mathrm{ppm}$ for 
${ }^{1} \mathrm{H}$ NMR and 77.16 ppm for ${ }^{13} \mathrm{C}$ NMR). Coupling constants $(J)$ are given in $\mathrm{Hz}$, multiplicities are abbreviated as: s (singlet), bs (broad singlet), d (doublet), dd (doublet of doublets), ddd (doublet of doublets of doublets), $\mathrm{t}$ (triplet), $\mathrm{m}$ (multiplet). High resolution mass spectra (HR-MS) were recorded on a LTQ-Orbitrap Mass Spectrometer [Thermo Scientific]. IR: Shimadzu IRAffinity-1CE spectrometer, wavenumbers in $\mathrm{cm}^{-1}$. Reactions were monitored by thin-layer chromatography carried out on silica plates (silica gel 60 F254, Merck) using UVlight for visualization. Column chromatography was performed on silica gel $60(0.040-0.063 \mathrm{~mm}$, Merck) using the indicated eluent given in volume ratio. All starting materials were purchased at the highest commercial quality and used without further purification unless otherwise stated.

Procedure A: General procedure for the synthesis of compounds 1a-c, 1e-j. The corresponding methylene active compound ( $1 \mathrm{mmol}, 1$ equiv) was added at r.t. to a solution of methyl coumalate (154 mg, $1 \mathrm{mmol}, 1$ equiv) in $\mathrm{EtOH}(5 \mathrm{~mL})$. After $\mathrm{Na}_{2} \mathrm{CO}_{3}$ (105 mg, $1 \mathrm{mmol}, 1$ equiv) was added to this colorless solution, it turned to orange. Thus prepared solution was stirred for $1 \mathrm{~h}$ at r.t. under Argon. Then the reaction mixture was diluted with $\mathrm{H}_{2} \mathrm{O}(20 \mathrm{~mL})$ and extracted with $\mathrm{CH}_{2} \mathrm{Cl}_{2}$ $(3 \times 20 \mathrm{~mL})$. Then the aqueous layer, containing the product as sodium salt, was acidified with $1 \mathrm{M} \mathrm{HCl}(5 \mathrm{~mL}$, until $\mathrm{pH}=2)$ and extracted with DCM $(3 \times 20 \mathrm{~mL})$. Collected organic layers were dried over anhydrous $\mathrm{MgSO}_{4}$, filtrated and evaporated under reduced pressure to afford the target products.

Procedure B: General procedure for the synthesis of compounds 2a-u. To a solution of methyl coumalate (154 mg, 1 mmol, 1 equiv) in $\mathrm{CH}_{2} \mathrm{Cl}_{2}(5 \mathrm{~mL})$ at r.t. was added the corresponding methylene active compound ( $1 \mathrm{mmol}, 1$ equiv) followed by triethylamine (101 $\mathrm{mg}, 1 \mathrm{mmol}, 1$ equiv). The solution was stirred for $15 \mathrm{~h}$ under reflux, cool to r.t. then quenched with $1 \mathrm{M} \mathrm{HCl}(10 \mathrm{~mL})$. The mixture was extracted with DCM $(3 \times 20 \mathrm{~mL})$. The organic layers were washed with brine and dried over anhydrous $\mathrm{MgSO}_{4}$, filtrated and evaporated and the residue was subjected to column chromatography on silica yielding the respective title compounds.

Procedure C: General procedure for the synthesis of compounds $3 \mathbf{d}, \mathbf{3 i}, \mathbf{3 j}$. The corresponding methylene active compound (1 mmol, 1 equiv) was added at r.t. to a solution of methyl coumalate (154 mg, $1 \mathrm{mmol}, 1$ equiv) in $\mathrm{EtOH}(5 \mathrm{~mL})$. After $\mathrm{Na}_{2} \mathrm{CO}_{3}$ (105 mg, $1 \mathrm{mmol}, 1$ equiv) was added to this colorless solution, it turned to orange. Thus prepared solution was stirred for $1 \mathrm{~h}$ at r.t. under Argon. Then N-(3dimethylaminopropyl)-N'-ethylcarbodiimide hydrochloride (192 mg, $1 \mathrm{mmol}, 1$ equiv) and DMAP (24 mg, $0.2 \mathrm{mmol}, 0.2$ equiv) was added in $\mathrm{CH}_{2} \mathrm{Cl}_{2}(5 \mathrm{~mL})$ solution, Once reaction was completed, the reaction mixture was quenched with $1 \mathrm{M}$ $\mathrm{HCl}(10 \mathrm{~mL})$ and mixture was extracted into $\mathrm{CH}_{2} \mathrm{Cl}_{2}(3 \times 20$ $\mathrm{mL})$ Collected organic layers were washed with brine and dried over anhydrous $\mathrm{MgSO}_{4}$, filtrated and evaporated and the residue was subjected to column chromatography on silica yielding the respective title compounds.

2-(3,5-bis(methoxycarbonyl)-6-methyl-2H-pyran-2-yl) acetic acid (1a): The title compound was prepared according to procedure A, $243 \mathrm{mg}$; 90\% yield; yellow oil; ${ }^{1} \mathbf{H}$ NMR (400 $\left.\mathrm{MHz}, \mathrm{CDCl}_{3}\right) \delta 10.65$ (br s, $\left.1 \mathrm{H}\right), 7.56(\mathrm{~s}, 1 \mathrm{H}), 5.73(\mathrm{dd}, J=$ $10.1,3.1 \mathrm{~Hz}, 1 \mathrm{H}), 3.77(\mathrm{~s}, 6 \mathrm{H}), 2.84(\mathrm{dd}, J=15.2,10.1 \mathrm{~Hz}$, $1 \mathrm{H}), 2.56(\mathrm{dd}, J=15.2,3.1 \mathrm{~Hz}, 1 \mathrm{H}), 2.37(\mathrm{~s}, 3 \mathrm{H}) ;{ }^{13} \mathbf{C} \mathbf{N M R}$ $\left(100 \mathrm{MHz}, \mathrm{CDCl}_{3}\right) \delta 174.9,170.6,165.4,164.6,132.3,114.8$, $104.4,72.2,51.9,51.6,37.9,20.2$; IR $\left(\right.$ film, $\left.\mathrm{cm}^{-1}\right)$ : $\sim 3600-$
2600 (br), 2976, 2868, 1716, 1436, 1362, 1244, 1182, 1112, 933, 790; HRMS (ESI) Found $m / z 293.0632$ calcd for $\left[\mathrm{M}+\mathrm{Na}^{+}\right]$ $\mathrm{C}_{12} \mathrm{H}_{14} \mathrm{NaO}_{7} 293.0632$.

2-(6-butyl-3,5-bis(methoxycarbonyl)-2H-pyran-2-yl)acetic acid (1b): The title compound was prepared according to procedure A, $268 \mathrm{mg}$; 90\% yield; yellow oil; ${ }^{1} \mathbf{H}$ NMR (400 $\left.\mathrm{MHz}, \mathrm{CDCl}_{3}\right) \delta 10.43$ (br s, $\left.1 \mathrm{H}\right), 7.57$ (s, 1H), 5.75 (dd, $J=$ $10.1,3.0 \mathrm{~Hz}, 1 \mathrm{H}), 3.77(\mathrm{~s}, 3 \mathrm{H}), 3.77(\mathrm{~s}, 3 \mathrm{H}), 2.98-2.76(\mathrm{~m}$, $2 \mathrm{H}), 2.55-2.57(\mathrm{~m}, 2 \mathrm{H}), 1.66-1.52(\mathrm{~m}, 2 \mathrm{H}), 0.95(\mathrm{t}, J=7.4$ $\mathrm{Hz}, 3 \mathrm{H}) ;{ }^{13} \mathrm{C}$ NMR $\left(100 \mathrm{MHz}, \mathrm{CDCl}_{3}\right) \delta 175.2,174.0,165.3$, 164.7, 132.6, 114.6, 104.1, 72.0, 51.9, 51.6, 37.7, 34.6, 20.7, 14.0; IR (film, $\mathrm{cm}^{-1}$ ): 3600-2600 (br), 2960, 2873, 1714, 1558, 1436, 1232, 1112, 1060, 977, 756; HRMS (ESI) Found $m / z 321.0957$ calcd for $\left[\mathrm{M}+\mathrm{Na}^{+}\right] \mathrm{C}_{14} \mathrm{H}_{18} \mathrm{NaO}_{7} 321.0945$

2-(3,5-bis (methoxycarbonyl) -6- (methoxymethyl)-2Hpyran-2-yl) acetic acid (1c): The title compound was prepared according to procedure A, $273 \mathrm{mg} ; 91 \%$ yield: colorless oil; ${ }^{1} \mathbf{H}$ NMR $\left(400 \mathrm{MHz}, \mathrm{CDCl}_{3}\right) \delta 9.63(\mathrm{br} \mathrm{s}, 1 \mathrm{H}), 7.53(\mathrm{~s}, 1 \mathrm{H})$, $5.83(\mathrm{dd}, \mathrm{J}=9.9,3.2 \mathrm{~Hz}, 1 \mathrm{H}), 4.65(\mathrm{~d}, J=14.0 \mathrm{~Hz}, 1 \mathrm{H}), 4.44$ $(\mathrm{d}, J=14.0 \mathrm{~Hz}, 1 \mathrm{H}), 3.79$ (s, 3H), 3.78 (s, 3H), 3.40 (s, 3H), $2.90(\mathrm{dd}, J=15.6,9.9 \mathrm{~Hz}, 1 \mathrm{H}), 2.57$ (dd, $J=15.6,3.2 \mathrm{~Hz}, 1 \mathrm{H})$; ${ }^{13}$ C NMR $\left(100 \mathrm{MHz}, \mathrm{CDCl}_{3}\right) \delta 173.9,167.4,164.8,164.3$, 131.5, 116.6, 105.9, 72.3, 69.3, 59.3, 52.1, 51.9, 37.6; IR (film, $\mathrm{cm}^{-1}$ ): 3700-2500 (br), 2953, 1710, 1641, 1570, 1242, 1112, 1064, 977, 756; HRMS (ESI) Found $\mathrm{m} / z 323.0748$ calcd for $\left[\mathrm{M}+\mathrm{Na}^{+}\right] \mathrm{C}_{13} \mathrm{H}_{16} \mathrm{NaO}_{8} 323.0737$.

2-(6-(chloromethyl)-3,5-bis(methoxycarbonyl)-2H-pyran-2yl) acetic acid (1e): The title compound was prepared according to procedure A , $255 \mathrm{mg} ; 84 \%$ yield; colorless oil; ${ }^{1} \mathbf{H}$ NMR $\left(400 \mathrm{MHz}, \mathrm{CDCl}_{3}\right) \delta 9.72($ br s, $1 \mathrm{H}), 7.52$ (s, 1H), 5.81 $(\mathrm{dd}, J=9.9,3.1 \mathrm{~Hz}, 1 \mathrm{H}), 4.90(\mathrm{~d}, J=11.3 \mathrm{~Hz}, 1 \mathrm{H}), 4.29$ (d, $J$ $=11.3 \mathrm{~Hz}, 1 \mathrm{H}), 3.81(\mathrm{~s}, 3 \mathrm{H}), 3.78(\mathrm{~s}, 3 \mathrm{H}), 2.93(\mathrm{dd}, J=16.2$, $9.9 \mathrm{~Hz}, 1 \mathrm{H}), 2.56(\mathrm{dd}, J=16.2,3.1 \mathrm{~Hz}, 1 \mathrm{H}) ;{ }^{13} \mathbf{C}$ NMR $(100$ $\left.\mathrm{MHz}, \mathrm{CDCl}_{3}\right) \delta 174.7,164.2,164.2,164.1,131.1,117.9,106.1$, 72.0, 52.2 (2C), 39.8, 37.4; IR (film, $\mathrm{cm}^{-1}$ ): 3700-2400 (br), 2978, 1716, 1436, 1384, 1251, 1238, 1193, 1180, 1097, 1070, 1002, 933, 833, 756; HRMS (ESI) Found m/z 303.0266, calcd for $\left[\mathrm{M}-\mathrm{H}^{+}\right] \mathrm{C}_{12} \mathrm{H}_{12} \mathrm{ClO}_{7} 303.0277$.

2-(6-(2-methoxy-2-oxoethyl)-3,5-bis(methoxycarbonyl)-2Hpyran-2-yl) acetic acid (1f): The title compound was prepared according to procedure A, $302 \mathrm{mg}$; $92 \%$ yield; colorless oil; ${ }^{1} \mathbf{H}$ NMR $\left(400 \mathrm{MHz}_{\mathrm{CDCl}}\right.$ ): $\delta 9.16(\mathrm{br} \mathrm{s}, 1 \mathrm{H}), 7.55(\mathrm{~s}, 1 \mathrm{H}) ; 5.80$ $(\mathrm{dd}, J=10.1,2.7 \mathrm{~Hz}, 1 \mathrm{H}) ; 4.14(\mathrm{~d}, J=16.2 \mathrm{~Hz}, 1 \mathrm{H}) ; 3.79(\mathrm{~s}$, $3 \mathrm{H}) ; 3.77$ (s, 3H); $3.71(\mathrm{~s}, 3 \mathrm{H}) ; 3.48\left(\mathrm{~d}, J=16.2 \mathrm{~Hz}, 1 \mathrm{H}^{\prime}\right) ; 3.07$ (dd, $J=16.4,10.1 \mathrm{~Hz}, 1 \mathrm{H}) ; 2.53(\mathrm{dd}, J=16.4,2.7 \mathrm{~Hz}, 1 \mathrm{H})$; ${ }^{13}$ C NMR $\left(100 \mathrm{MHz}, \mathrm{CDCl}_{3}\right) \delta 174.8 ; 168.7 ; 164.9 ; 164.6$; $164.4 ; 131.4 ; 116.5 ; 106.4 ; 72.4 ; 52.6 ; 52.1 ; 52.0 ; 39.0 ; 37.4$; IR (film, cm ${ }^{-1}$ ): 3500-2400 (br), 2960, 1714, 1697, 11435, 1357, 1247, 1093, 997, 877, 775; HRMS (ESI) Found $m / z$ 351.0687, calcd for $\left[\mathrm{M}+\mathrm{Na}^{+}\right] \mathrm{C}_{14} \mathrm{H}_{16} \mathrm{NaO}_{9} 351.0691$.

2-(5-acetyl-3-(methoxycarbonyl)-6-methyl-2H-pyran-2yl)acetic acid (1g): The title compound was prepared according to procedure A, $228 \mathrm{mg}$; 90\% yield; colorless oil; ${ }^{1} \mathbf{H}$ NMR $\left(400 \mathrm{MHz}, \mathrm{CDCl}_{3}\right) \delta 10.42$ (br s, $\left.1 \mathrm{H}\right), 7.46(\mathrm{~s}, 1 \mathrm{H}), 5.74-5.54$ $(\mathrm{m}, 1 \mathrm{H}), 3.73(\mathrm{~s}, 3 \mathrm{H}), 2.77(\mathrm{~m}, 1 \mathrm{H}), 2.50(\mathrm{~m}, 1 \mathrm{H}), 2.28(\mathrm{~s}, 6 \mathrm{H})$; ${ }^{13}$ C NMR $\left(100 \mathrm{MHz}, \mathrm{CDCl}_{3}\right) \delta 195.6,174.4,171.1,164.5$, 132.3, 114.8, 112.9, 72.3, 52.0, 37.9, 28.9, 21.0; IR (film, $\mathrm{cm}^{-}$ $\left.{ }^{1}\right)$ : 3500-2400 (br), 2976, 2870, 1712, 1624, 1436, 1382, 1238, $1105,1070,1018,935,840$; HRMS (ESI) Found $\mathrm{m} / \mathrm{z}$ 277.0784, calcd for $\left[\mathrm{M}+\mathrm{Na}^{+}\right] \mathrm{C}_{12} \mathrm{H}_{14} \mathrm{NaO}_{6} 277.0683$.

Dimethyl-2,6-dimethyl-2H-pyran-3,5-dicarboxylate (2a): The title compound was prepared according to procedure $\mathrm{B}$, 
$196 \mathrm{mg} ; 87 \%$ yield; colorless oil; $\mathrm{R}_{\mathrm{f}}=0.2[1: 5(\mathrm{v} / \mathrm{v})$ EA/cyclohexane]; ${ }^{1} \mathbf{H}$ NMR $\left(400 \mathrm{MHz}, \mathrm{CDCl}_{3}\right) \delta 7.47(\mathrm{~s}, 1 \mathrm{H})$, $5.34(\mathrm{q}, \mathrm{J}=6.5 \mathrm{~Hz}, 1 \mathrm{H}), 3.74(\mathrm{~s}, 3 \mathrm{H}), 3.74(\mathrm{~s}, 3 \mathrm{H}), 2.34(\mathrm{~s}, 3 \mathrm{H})$, $1.30(\mathrm{~d}, J=6.5 \mathrm{~Hz}, 3 \mathrm{H}) ;{ }^{13} \mathbf{C}$ NMR $\left(100 \mathrm{MHz}, \mathrm{CDCl}_{3}\right) \delta 170.8$, $165.8,165.0,130.8,117.2,103.5,72.5,51.6,51.4,20.5,19.2$; IR (film, $\mathrm{cm}^{-1}$ ): 2951, 2918, 1716, 1689, 1637, 1433, 1379, 1309, 1226, 1128, 1045, 910, 752; HRMS (ESI) Found $m / z$ 233.0996, calcd for $\left[\mathrm{M}+\mathrm{Li}^{+}\right] \mathrm{C}_{11} \mathrm{H}_{14} \mathrm{LiO}_{5} 233.0996$.

Dimethyl-2-methyl-6-propyl-2H-pyran-3,5-dicarboxylate (2b): The title compound was prepared according to procedure B, $224 \mathrm{mg} ; 88 \%$ yield; white solid, m.p.123-126 ${ }^{\circ} \mathrm{C} ; \mathrm{R}_{\mathrm{f}}=0.2$ [1:5 (v/v) EA/cyclohexane]; ${ }^{1} \mathbf{H}$ NMR (400 $\left.\mathrm{MHz}, \mathrm{CDCl}_{3}\right) \delta$ $7.47(\mathrm{~s}, 1 \mathrm{H}), 5.34(\mathrm{q}, J=6.5 \mathrm{~Hz}, 1 \mathrm{H}), 3.73(\mathrm{~s}, 3 \mathrm{H}), 3.73(\mathrm{~s}$, $3 \mathrm{H}), 3.04-2.84(\mathrm{~m}, 1 \mathrm{H}), 2.51-242(\mathrm{~m}, 1 \mathrm{H}), 1.67-1.50(\mathrm{~m}$, $2 \mathrm{H}), 1.27(\mathrm{~d}, J=6.5 \mathrm{~Hz}, 3 \mathrm{H}), 0.95(\mathrm{t}, J=7.4 \mathrm{~Hz}, 3 \mathrm{H}) ;{ }^{13} \mathbf{C}$ NMR $\left(100 \mathrm{MHz}, \mathrm{CDCl}_{3}\right) \delta 174.1,165.6,165.0,131.0,117.2$, 103.4, 72.2, 51.6, 51.3, 34.7, 20.6, 19.0, 13.9; IR (film, cm ${ }^{-1}$ ): 2974, 2872, 1701, 1639, 1556, 1435, 1307, 1224, 1122, 1068, 914, 756; HRMS (ESI) Found $\mathrm{m} / \mathrm{z}$ 277.1051, calcd for $\left[\mathrm{M}+\mathrm{Na}^{+}\right] \mathrm{C}_{13} \mathrm{H}_{18} \mathrm{NaO}_{5} 277.1046$

Dimethyl-6-(methoxymethyl)-2-methyl-2H-pyran-3,5dicarboxylate (2c): The title compound was prepared according to procedure B, $208 \mathrm{mg}, 81 \%$ yield, white solid, m.p.140$141^{\circ} \mathrm{C}, \mathrm{R}_{\mathrm{f}}=0.3[1: 5(\mathrm{v} / \mathrm{v}) \mathrm{EA} /$ cyclohexane $] ;{ }^{1} \mathbf{H}$ NMR $(400$ $\left.\mathrm{MHz}, \mathrm{CDCl}_{3}\right) \delta 7.44(\mathrm{~s}, 1 \mathrm{H}), 5.46(\mathrm{q}, J=6.5 \mathrm{~Hz}, 1 \mathrm{H}), 4.67(\mathrm{~d}$, $J=14.3 \mathrm{~Hz}, 1 \mathrm{H}), 4.40$ (d, $J=14.3 \mathrm{~Hz}, 1 \mathrm{H}), 3.76$ (s, 3H), 3.75 $(\mathrm{s}, 3 \mathrm{H}), 3.39(\mathrm{~s}, 3 \mathrm{H}), 1.35(\mathrm{~d}, J=6.5 \mathrm{~Hz}, 3 \mathrm{H}) ;{ }^{13} \mathbf{C}$ NMR $(100$ $\left.\mathrm{MHz}, \mathrm{CDCl}_{3}\right) \delta 168.0,165.1,164.7,130.1,118.8,104.9,72.8$, 69.6, 59.1, 51.8, 51.6, 19.0; IR (film, $\mathrm{cm}^{-1}$ ): 2951, 2931, 1699, $1639,1566,1435,1309,1226,1124,1076,991,912,752$; HRMS (ESI) Found $\mathrm{m} / \mathrm{z} 279.0835$ calcd for $\left[\mathrm{M}+\mathrm{Na}^{+}\right]$ $\mathrm{C}_{12} \mathrm{H}_{16} \mathrm{NaO}_{6} 279.0839$.

Dimethyl-6-cyclopropyl-2-methyl-2H-pyran-3,5-

dicarboxylate (2d): The title compound was prepared according to procedure B, $237 \mathrm{mg}$; 94\% yield; white solid, m.p.101$103^{\circ} \mathrm{C} ; \mathrm{R}_{\mathrm{f}}=0.2[1: 5(\mathrm{v} / \mathrm{v}) \mathrm{EA} /$ cyclohexane $] ;{ }^{1} \mathbf{H}$ NMR $(400$ $\left.\mathrm{MHz} \mathrm{CDCl}_{3}\right) \delta 7.52(\mathrm{~s}, 1 \mathrm{H}), 5.27(\mathrm{q}, J=6.5 \mathrm{~Hz}, 1 \mathrm{H}), 3.77(\mathrm{~s}$, $3 \mathrm{H}), 3.73(\mathrm{~s}, 3 \mathrm{H}), 3.17-3.10(\mathrm{~m}, 1 \mathrm{H}), 1.35-1.24(\mathrm{~m}, 1 \mathrm{H})$, $1.21(\mathrm{~d}, J=6.5 \mathrm{~Hz}, 3 \mathrm{H}), 1.09-0.97(\mathrm{~m}, 1 \mathrm{H}), 0.97-0.88(\mathrm{~m}$, $1 \mathrm{H}), 0.88-0.74(\mathrm{~m}, 1 \mathrm{H}) ;{ }^{13} \mathbf{C}$ NMR $\left(100 \mathrm{MHz}, \mathrm{CDCl}_{3}\right) \delta$ 173.8, 166.2, 165.1, 131.4, 116.3, 103.5, 72.0, 51.5, 51.4, 18.6, 13.1, 10.1, 7.1; IR (film, $\mathrm{cm}^{-1}$ ): 2951, 2929, 1687, 1635, 1539, 1301, 1222, 1062, 1043, 931, 875, 815; HRMS (ESI) Found $m / z 275.0885$, calcd for $\left[\mathrm{M}+\mathrm{Na}^{+}\right] \mathrm{C}_{13} \mathrm{H}_{16} \mathrm{NaO}_{5} 275.0890$.

Dimethyl-6-(chloromethyl)-2-methyl-2H-pyran-3,5dicarboxylate (2e): The title compound was prepared according to procedure $\mathrm{B}, 179 \mathrm{mg} ; 71 \%$ yield; colorless oil; $\mathrm{R}_{\mathrm{f}}=0.2$ [1:5 (v/v) EA/cyclohexane]; ${ }^{1} \mathbf{H}$ NMR (400 $\left.\mathrm{MHz}, \mathrm{CDCl}_{3}\right) \delta$ $7.45(\mathrm{~d}, J=0.4 \mathrm{~Hz}, 1 \mathrm{H}), 5.43$ (q, $J=6.5 \mathrm{~Hz}, 1 \mathrm{H}), 4.97$ (d, $J=$ $11.2 \mathrm{~Hz}, 1 \mathrm{H}), 4.24$ (d, $J=11.2 \mathrm{~Hz}, 1 \mathrm{H}), 3.81(\mathrm{~s}, 3 \mathrm{H}), 3.78$ (s, $3 \mathrm{H}), 1.36(\mathrm{~d}, J=6.5 \mathrm{~Hz}, 3 \mathrm{H}) ;{ }^{13} \mathbf{C}$ NMR $\left(100 \mathrm{MHz}, \mathrm{CDCl}_{3}\right) \delta$ 164.6, 164.1, 129.6, 120.4, 105.5, 72.6, 52.0, 51.9, 40.1, 18.9; IR (film, $\mathrm{cm}^{-1}$ ): 2953, 2926, 1703; 1643, 1573, 1435, 1307, 1228, 1124, 1076, 939, 796, 754; HRMS (ESI) Found $\mathrm{m} / \mathrm{z}$ 267.0610, calcd for $\left[\mathrm{M}+\mathrm{Li}^{+}\right] \mathrm{C}_{11} \mathrm{H}_{13} \mathrm{ClLiO}_{5}$ 267.0606.

Dimethyl-6-(2-methoxy-2-oxoethyl)-2-methyl-2H-pyran3,5-dicarboxylate (2f): The title compound was prepared according to procedure $\mathrm{B}, 233 \mathrm{mg}, 82 \%$ yield, colorless oil, $\mathrm{R}_{\mathrm{f}}$ $=0.2[1: 5(\mathrm{v} / \mathrm{v}) \mathrm{EA} /$ cyclohexane $] ;{ }^{1} \mathbf{H} \mathbf{~ N M R}\left(400 \mathrm{MHz}, \mathrm{CDCl}_{3}\right)$ $\delta 7.41(\mathrm{~s}, 1 \mathrm{H}), 5.35(\mathrm{q}, J=6.5 \mathrm{~Hz}, 1 \mathrm{H}), 4.05(\mathrm{~d}, J=16.2 \mathrm{~Hz}$, $1 \mathrm{H}), 3.71(\mathrm{~s}, 3 \mathrm{H}), 3.71(\mathrm{~s}, 3 \mathrm{H}), 3.67(\mathrm{~s}, 3 \mathrm{H}), 3.44(\mathrm{~d}, J=16.2$ $\mathrm{Hz}, 1 \mathrm{H}), 1.32(\mathrm{~d}, J=6.5 \mathrm{~Hz}, 3 \mathrm{H}) ;{ }^{13} \mathbf{C}$ NMR $(100 \mathrm{MHz}$
$\left.\mathrm{CDCl}_{3}\right) \delta 168.7,165.2,164.8,164.7,129.8,118.8,105.4,73.0$, 52.2, 51.7, 51.6, 39.0, 19.1; IR (film, $\mathrm{cm}^{-1}$ ): 3001, 2954, 1739, 1712, 1689, 1641, 1573, 1463, 1332, 1122, 1047, 948, 871; HRMS (ESI) Found $m / z$ 307.0784, calcd for $\left[\mathrm{M}+\mathrm{Na}^{+}\right]$ $\mathrm{C}_{13} \mathrm{H}_{16} \mathrm{NaO}_{7} 307.0788$.

Methyl-5-acetyl-2,6-dimethyl-2H-pyran-3-carboxylate (2g): The title compound was prepared according to procedure B, $199 \mathrm{mg} ; 95 \%$ yield, colorless oil, $\mathrm{R}_{\mathrm{f}}=0.2[1: 5(\mathrm{v} / \mathrm{v})$ EA/cyclohexane]; ${ }^{1} \mathbf{H}$ NMR $\left(400 \mathrm{MHz}, \mathrm{CDCl}_{3}\right) \delta 7.40(\mathrm{~s}, 1 \mathrm{H})$, $5.32(\mathrm{q}, J=6.5 \mathrm{~Hz}, 1 \mathrm{H}), 3.73(\mathrm{~s}, 3 \mathrm{H}), 2.29(\mathrm{~s}, 3 \mathrm{H}), 2.28(\mathrm{~s}$, $3 \mathrm{H}), 1.27(\mathrm{~d}, J=6.5 \mathrm{~Hz}, 3 \mathrm{H}) ;{ }^{13} \mathbf{C} \mathbf{N M R}\left(100 \mathrm{MHz}, \mathrm{CDCl}_{3}\right) \delta$ 195.2, 171.1, 164.9, 131.0, 117.1, 112.3, 72.5, 51.6, 28.9, 21.3, 19.2; IR (film, $\mathrm{cm}^{-1}$ ): 2976, 1703, 1633, 1558, 1436, 1274, 1228, 1147, 1122, 954, 756; HRMS (ESI) Found $\mathrm{m} / \mathrm{z}$ 233.0783 calcd for $\left[\mathrm{M}+\mathrm{Na}^{+}\right] \mathrm{C}_{11} \mathrm{H}_{14} \mathrm{NaO}_{4} 233.0784$.

Methyl-5-acetyl-2-methyl-6-phenyl-2H-pyran-3-

carboxylate (2h): The title compound was prepared according to procedure $\mathrm{B}, 117 \mathrm{mg}, 43 \%$ yield, yellow oil, $\mathrm{R}_{\mathrm{f}}=0.2[1: 5$ (v/v) EA/cyclohexane]; ${ }^{1} \mathbf{H}$ NMR $\left(400 \mathrm{MHz}, \mathrm{CDCl}_{3}\right) \delta 7.69$ (s, $1 \mathrm{H}), 7.60-7.30(\mathrm{~m}, 5 \mathrm{H}), 5.53(\mathrm{q}, J=6.5 \mathrm{~Hz}, 1 \mathrm{H}), 3.78(\mathrm{~s}, 3 \mathrm{H})$, $1.88(\mathrm{~s}, 3 \mathrm{H}), 1.48(\mathrm{~d}, J=6.5 \mathrm{~Hz}, 3 \mathrm{H}) ;{ }^{13} \mathrm{C}$ NMR $(100 \mathrm{MHz}$, $\left.\mathrm{CDCl}_{3}\right) \delta 196.8,165.6,165.0,133.7,131.7,131.1,129.9$, 128.5, 119.0, 115.8, 72.6, 51.8, 29.8, 18.3; IR (film, $\mathrm{cm}^{-1}$ ): 2976, 1705, 1625, 1435, 1363, 1283, 1119, 875, 771, 793; HRMS (ESI) Found $\mathrm{m} / z$ 295.0942, calcd for $\left[\mathrm{M}+\mathrm{Na}^{+}\right]$ $\mathrm{C}_{16} \mathrm{H}_{16} \mathrm{NaO}_{4} 295.0941$.

\section{Methyl-5-benzoyl-2,6-dimethyl-2H-pyran-3-carboxylate} (2h'): The title compound was prepared according to procedure $\mathrm{B}, 106 \mathrm{mg}, 39 \%$ yield, colorless oil, $\mathrm{R}_{\mathrm{f}}=0.3[1: 5(\mathrm{v} / \mathrm{v})$ EA/cyclohexane]; ${ }^{1} \mathbf{H}$ NMR $\left(400 \mathrm{MHz}, \mathrm{CDCl}_{3}\right) \delta 7.72-7.38$ $(\mathrm{m}, 5 \mathrm{H}), 7.32(\mathrm{~s}, 1 \mathrm{H}), 5.43(\mathrm{q}, J=6.5 \mathrm{~Hz}, 1 \mathrm{H}), 3.72(\mathrm{~s}, 3 \mathrm{H})$, $2.09(\mathrm{~s}, 3 \mathrm{H}), 1.44(\mathrm{~d}, J=6.5 \mathrm{~Hz}, 3 \mathrm{H}) ;{ }^{13} \mathbf{C}$ NMR $(100 \mathrm{MHz}$, $\left.\mathrm{CDCl}_{3}\right) \delta 193.7,169.2,165.1,139.1,132.3,131.6,128.9$, 128.6, 116.8, 112.2, 72.4, 51.6, 26.9, 20.7, 19.3; IR (film, $\mathrm{cm}^{-}$ $\left.{ }^{1}\right)$ : 2976, 1703, 1624, 1435, 1381, 1238, 1147, 1043, 939, 877, 725; HRMS (ESI) Found $m / z$ 311.0675, calcd for $\left[\mathrm{M}+\mathrm{K}^{+}\right]$ $\mathrm{C}_{16} \mathrm{H}_{16} \mathrm{KO}_{4} 311.0680$.

Methyl-2-methyl-5-oxo-5,6,7,8-tetrahydro-2H-chromene-3carboxylate (2i): The title compound was prepared according to procedure $\mathrm{B}, 188 \mathrm{mg}, 85 \%$ yield, colorless oil, $\mathrm{R}_{\mathrm{f}}=0.3[1: 5$ (v/v) EA/cyclohexane]; ${ }^{1} \mathbf{H}$ NMR $\left(400 \mathrm{MHz}, \mathrm{CDCl}_{3}\right) \delta 7.50(\mathrm{~s}$, $1 \mathrm{H}), 5.39(\mathrm{q}, J=6.5 \mathrm{~Hz}, 1 \mathrm{H}), 3.72(\mathrm{~s}, 3 \mathrm{H}), 2.49-2.43(\mathrm{~m}, 2 \mathrm{H})$, $2.43-2.36(\mathrm{~m}, 2 \mathrm{H}), 2.05-1.88(\mathrm{~m}, 2 \mathrm{H}), 1.33(\mathrm{~d}, J=6.5 \mathrm{~Hz}$, $3 \mathrm{H}) ;{ }^{13} \mathrm{C}$ NMR $\left(100 \mathrm{MHz}, \mathrm{CDCl}_{3}\right) \delta 194.1,175.0,165.1$, 126.7, 119.4, 110.9, 73.5, 51.6, 36.4, 28.8, 20.3, 20.2; IR (film, $\left.\mathrm{cm}^{-1}\right): 2954,2927,1703,1633,1556,1435,1381,1274,1145$, 1122, 1076, 954, 754; HRMS (ESI) Found $\mathrm{m} / \mathrm{z} 245.0789$, calcd for $\left[\mathrm{M}+\mathrm{Na}^{+}\right] \mathrm{C}_{12} \mathrm{H}_{14} \mathrm{NaO}_{4} 245.0784$.

Methyl-2,6-dimethyl-5-(methylsulfonyl)-2H-pyran-3carboxylate $(\mathbf{2 j})$ : The title compound was prepared according to procedure $\mathrm{B}, 223 \mathrm{mg}, 91 \%$ yield, colorless oil, $\mathrm{R}_{\mathrm{f}}=0.2[1: 5$ (v/v) EA/cyclohexane]; ${ }^{1} \mathbf{H}$ NMR $\left(400 \mathrm{MHz}, \mathrm{CDCl}_{3}\right) \delta 7.28(\mathrm{~s}$, 1H), 5.36 (q, $J=6.5 \mathrm{~Hz}, 1 \mathrm{H}), 3.73(\mathrm{~s}, 3 \mathrm{H}), 2.95(\mathrm{~s}, 3 \mathrm{H}), 2.33$ $(\mathrm{s}, 3 \mathrm{H}), 1.34(\mathrm{~d}, J=6.5 \mathrm{~Hz}, 3 \mathrm{H}) ;{ }^{13} \mathbf{C}$ NMR $\left(100 \mathrm{MHz}, \mathrm{CDCl}_{3}\right)$ $\delta 168.0,164.3,128.0$ (2C), 118.4, 113.8, 73.1, 51.9, 44.2, 19.3; IR (film, $\mathrm{cm}^{-1}$ ): 2980, 2954, 1703, 1633, 1556, 1435, 1381, 1274, 1228, 1145, 1122, 1043, 954, 754; HRMS (ESI) Found $m / z 269.0458$, calcd for $\left[\mathrm{M}+\mathrm{Na}^{+}\right] \mathrm{C}_{10} \mathrm{H}_{14} \mathrm{NaO}_{5} \mathrm{~S} 269.0454$.

Dimethyl-6-(tert-butyl)-2-methyl-2H-pyran-3,5dicarboxylate (2k): The title compound was prepared according to Procedure $\mathrm{B}, 71 \mathrm{mg}, 27 \%$ yield, colorless oil, $\mathrm{R}_{\mathrm{f}}=0.2$ [1:5 (v/v) EA/cyclohexane]; ${ }^{1} \mathbf{H}$ NMR (400 $\left.\mathrm{MHz} \mathrm{CDCl}_{3}\right) \delta$ 
$7.36(\mathrm{~s}, 1 \mathrm{H}), 5.36(\mathrm{q}, J=6.5 \mathrm{~Hz}, 1 \mathrm{H}), 3.76(\mathrm{~s}, 3 \mathrm{H}), 3.75(\mathrm{~s}$, $3 \mathrm{H}), 1.27(\mathrm{~d}, J=6.5 \mathrm{~Hz}, 3 \mathrm{H}), 1.25(\mathrm{~s}, 9 \mathrm{H}) ;{ }^{13} \mathbf{C}$ NMR $(100$ $\left.\mathrm{MHz}, \mathrm{CDCl}_{3}\right) \delta 174.8,167.4,164.9,132.7,116.6,105.2,71.3$, 51.7, 51.6, 38.2, 27.6, 17.5; IR (film, cm ${ }^{-1}$ ): 2951, 1701,1637, 1529, 1435, 1384, 1301, 1230, 1215, 1107, 933, 914, 756; HRMS (ESI) Found $m / z, 291.1214$, calcd for $\left[\mathrm{M}+\mathrm{Na}^{+}\right]$ $\mathrm{C}_{14} \mathrm{H}_{20} \mathrm{NaO}_{5} 291.1203$

5-ethyl-3-methyl-2-methyl-6-phenyl-2H-pyran-3,5dicarboxylate (21): The title compound was prepared according to procedure $\mathrm{B}, 262 \mathrm{mg}, 80 \%$ yield, yellow oil, $\mathrm{R}_{\mathrm{f}}=0.2$ [1:5 (v/v) EA/cyclohexane]; ${ }^{1} \mathbf{H}$ NMR (400 $\left.\mathrm{MHz}, \mathrm{CDCl}_{3}\right) \delta$ $7.65(\mathrm{~s}, 1 \mathrm{H}), 7.53-7.30(\mathrm{~m}, 5 \mathrm{H}), 5.53(\mathrm{q}, J=6.5 \mathrm{~Hz}, 1 \mathrm{H})$, 4.07 (q, $J=7.1,2 \mathrm{H}), 3.78(\mathrm{~s}, 3 \mathrm{H}), 1.49(\mathrm{~d}, J=6.5 \mathrm{~Hz}, 3 \mathrm{H})$, $1.05(\mathrm{t}, J=7.1 \mathrm{~Hz}, 3 \mathrm{H}) ;{ }^{13} \mathbf{C}$ NMR $\left(100 \mathrm{MHz}, \mathrm{CDCl}_{3}\right) \delta 165.8$, 165.7, 164.9, 133.9, 131.6, 130.8(2C), 129.5(2C), 127.7, 118.5, 105.3, 72.7, 60.4, 51.7, 18.5, 13.8; IR (film, $\mathrm{cm}^{-1}$ ): 2978, 1697, $1637,1548,1487,1436,1375,1300,1230,1124,1080,989$, 920, 754; HRMS (ESI) Found $\mathrm{m} / \mathrm{z}$ 325.1071, calcd for $\left[\mathrm{M}+\mathrm{Na}^{+}\right] \mathrm{C}_{17} \mathrm{H}_{18} \mathrm{NaO}_{5} 325.1052$.

5-ethyl-3-methyl-2-methyl-6-(4-nitrophenyl)-2H-pyran-3,5dicarboxylate (2m): The title compound was prepared according to procedure $\mathrm{B}, 336 \mathrm{mg}, 97 \%$ yield, yellow oil, $\mathrm{R}_{\mathrm{f}}=0.2$ [1:5 (v/v) EA/cyclohexane]; ${ }^{1} \mathbf{H}$ NMR (400 $\left.\mathrm{MHz}, \mathrm{CDCl}_{3}\right) \delta$ $8.24(\mathrm{~d}, J=8.9 \mathrm{~Hz}, 2 \mathrm{H}), 7.62(\mathrm{~s}, 1 \mathrm{H}), 7.61(\mathrm{~d}, J=8.9 \mathrm{~Hz}, 2 \mathrm{H})$, $5.56(\mathrm{q}, J=6.5 \mathrm{~Hz}, 1 \mathrm{H}), 4.11(\mathrm{q}, J=7.1 \mathrm{~Hz}, 2 \mathrm{H}), 3.82(\mathrm{~s}, 3 \mathrm{H})$, $1.52(\mathrm{~d}, J=6.5 \mathrm{~Hz}, 3 \mathrm{H}), 1.12(\mathrm{t}, J=7.1 \mathrm{~Hz}, 3 \mathrm{H}) ;{ }^{13} \mathbf{C} \mathbf{N M R}$ $\left(100 \mathrm{MHz}, \mathrm{CDCl}_{3}\right) \delta 164.7,164.6,163.0,148.8,140.1,130.6$, $130.5,123.0,120.0,106.7,73.1,60.9,52.0,18.9,13.9$; IR (film, $\mathrm{cm}^{-1}$ ): 2989, 1693, 1633, 1546, 1435, 1371, 1298, 1236, 1134, 1018, 1991, 856, 752; HRMS (ESI) Found $\mathrm{m} / \mathrm{z}$ 370.0910, calcd for $\left[\mathrm{M}+\mathrm{Na}^{+}\right] \mathrm{C}_{17} \mathrm{H}_{17} \mathrm{NNaO}_{7} 370.0903$.

Methyl-2-methyl-5-oxo-2,5-dihydropyrano-[3,2-c]chromene-3-carboxylate (2n): The title compound was prepared according to procedure B, $222 \mathrm{mg}, 82 \%$ yield, yellow oil; $\mathrm{R}_{\mathrm{f}}=0.2[1: 5(\mathrm{v} / \mathrm{v})$ EA/cyclohexane $] ;{ }^{1} \mathbf{H} \mathbf{N M R}(400 \mathrm{MHz}$, $\left.\mathrm{CDCl}_{3}\right) \delta 7.86-7.81(\mathrm{~m}, 1 \mathrm{H}), 7.66(\mathrm{~s}, 1 \mathrm{H}), 7.64-7.55(\mathrm{~m}$, $1 \mathrm{H}), 7.36-7.28(\mathrm{~m}, 2 \mathrm{H}), 5.71(\mathrm{q}, J=6.5 \mathrm{~Hz}, 1 \mathrm{H}), 3.82(\mathrm{~s}, 3 \mathrm{H})$, $1.50(\mathrm{~d}, J=6.5 \mathrm{~Hz}, 3 \mathrm{H}) ;{ }^{13} \mathbf{C} \mathbf{N M R}\left(100 \mathrm{MHz}, \mathrm{CDCl}_{3}\right) \delta 164.5$, $161.4,160.1,153.9,133.7,127.4,124.4,123.6,122.3,117.1$, 114.8, 100.4, 74.0, 52.0, 20.5; IR (film, cm-1): 2926, 2854, 1722, 1699, 1637, 1606, 1556, 1490, 1296, 1242, 1205, 1029, 975, 935, 752; HRMS (ESI) Found $\mathrm{m} / z$ 295.0584, calcd for $\left[\mathrm{M}+\mathrm{Na}^{+}\right] \mathrm{C}_{15} \mathrm{H}_{12} \mathrm{NaO}_{5} 295.0577$.

Methyl-6-ethyl-2-methyl-5-propionyl-2H-pyran-3-

carboxylate (2o): The title compound was prepared according to procedure $\mathrm{B}, 216 \mathrm{mg}, 91 \%$ yield, colorless oil; $\mathrm{R}_{\mathrm{f}}=0.2[1: 5$ (v/v) EA/cyclohexane]; ${ }^{1} \mathbf{H}$ NMR $\left(400 \mathrm{MHz}, \mathrm{CDCl}_{3}\right) \delta 7.42(\mathrm{~s}$, $1 \mathrm{H}), 5.32(\mathrm{q}, J=6.5 \mathrm{~Hz}, 1 \mathrm{H}), 3.72(\mathrm{~s}, 3 \mathrm{H}), 2.98-2.77(\mathrm{~m}, 1 \mathrm{H})$, $2.73-2.50(\mathrm{~m}, 2 \mathrm{H}), 2.48-2.29(\mathrm{~m}, 1 \mathrm{H}), 1.25(\mathrm{~d}, J=6.5 \mathrm{~Hz}$, $3 \mathrm{H}), 1.10(\mathrm{t}, J=7.5 \mathrm{~Hz}, 3 \mathrm{H}), 1.06(\mathrm{t}, J=7.2 \mathrm{~Hz}, 3 \mathrm{H}) ;{ }^{13} \mathbf{C}$ NMR $\left(100 \mathrm{MHz}, \mathrm{CDCl}_{3}\right) \delta 197.8,174.7,164.9,130.7,117.1$, 111.4, 71.9, 51.6, 33.5, 26.9, 18.7, 11.1, 8.1. IR (film, cm-1): 2980, 2941, 1707, 1678, 1624, 1531, 1436, 1377, 1290, 1220, 1195, 1068, 898, 761; HRMS (ESI) Found $\mathrm{m} / \mathrm{z} 277.0840$, calcd for $\left[\mathrm{M}+\mathrm{K}^{+}\right] \mathrm{C}_{13} \mathrm{H}_{18} \mathrm{KO}_{4} 277.0837$.

Methyl-5-benzoyl-2-methyl-6-phenyl-2H-pyran-3-

carboxylate (2p): The title compound was prepared according to procedure $\mathrm{B}, 166 \mathrm{mg}, 50 \%$ yield, yellow oil, $\mathrm{R}_{\mathrm{f}}=0.3[1: 5$ (v/v) EA/cyclohexane]; ${ }^{1} \mathbf{H}$ NMR $\left(400 \mathrm{MHz}, \mathrm{CDCl}_{3}\right) \delta 7.64(\mathrm{~s}$, $1 \mathrm{H}), 7.53-7.46(\mathrm{~m}, 2 \mathrm{H}), 7.26-7.14(\mathrm{~m}, 3 \mathrm{H}), 7.15-6.97(\mathrm{~m}$, $5 \mathrm{H}), 5.57(\mathrm{q}, J=6.5 \mathrm{~Hz}, 1 \mathrm{H}), 3.73(\mathrm{~s}, 3 \mathrm{H}), 1.57(\mathrm{~d}, J=6.5 \mathrm{~Hz}$, $3 \mathrm{H}) ;{ }^{13} \mathbf{C}$ NMR (100 MHz, $\left.\mathrm{CDCl}_{3}\right) \delta 193.9,165.0,164.0$,
$138.1,133.0,132.2,132.1,131.1,130.1(2 \mathrm{C}), 129.3(2 \mathrm{C})$, 128.0(2C), 127.9(2C), 119.0, 113.4, 72.4, 51.8, 18.6; IR (film, cm-1): 2974, 2852, 1695, 1672, 1543, 1446, 1435, 1240, 1176, 1053, 1020 941, 904, 771; HRMS (ESI) Found $m / z$ 357.1107, calcd for $\left[\mathrm{M}+\mathrm{Na}^{+}\right] \mathrm{C}_{21} \mathrm{H}_{18} \mathrm{NaO}_{4} 357.1097$.

Methyl-2,7,7-trimethyl-5-oxo-5,6,7,8-tetrahydro-2Hchromene-3-carboxylate (2q): The title compound was prepared according to procedure B, $210 \mathrm{mg}, 84 \%$ yield, colorless oil, $\mathrm{R}_{\mathrm{f}}=0.2\left[1: 5(\mathrm{v} / \mathrm{v})\right.$ EA/cyclohexane]; ${ }^{1} \mathbf{H}$ NMR $(400 \mathrm{MHz}$, $\left.\mathrm{CDCl}_{3}\right) \delta 7.51(\mathrm{~s}, 1 \mathrm{H}), 5.41(\mathrm{q}, J=6.5 \mathrm{~Hz}, 1 \mathrm{H}), 3.72(\mathrm{~s}, 3 \mathrm{H})$, $2.43-2.31(\mathrm{~m}, 2 \mathrm{H}), 2.30-2.11(\mathrm{~m}, 2 \mathrm{H}), 1.33(\mathrm{~d}, J=6.5 \mathrm{~Hz}$, $3 \mathrm{H}), 1.08(\mathrm{~s}, 3 \mathrm{H}), 1.00(\mathrm{~s}, 3 \mathrm{H}) ;{ }^{13} \mathbf{C}$ NMR $\left(100 \mathrm{MHz}, \mathrm{CDCl}_{3}\right) \delta$ 193.8, 173.7, 165.1, 126.6, 119.1, 109.9, 73.6, 51.6, 50.3, 42.5, 32.3, 29.4, 27.2, 20.2; IR (film, cm-1): 2965, 2872, 1703, 1660, 1571, 1398, 1307, 1217, 1145, 1068, 939, 761; HRMS (ESI) Found $m / z$ 273.1099, calcd for $\left[\mathrm{M}+\mathrm{Na}^{+}\right] \mathrm{C}_{14} \mathrm{H}_{18} \mathrm{NaO}_{4} 273.1097$.

Methyl-2-methyl-5-oxo-2,5,6,7-tetrahydrocyclopenta-[b]pyran-3-carboxylate (2r): The title compound was prepared according to procedure $\mathrm{B}, 201 \mathrm{mg}, 96 \%$ yield, colorless oil, $\mathrm{R}_{\mathrm{f}}$ $=0.2[1: 5(\mathrm{v} / \mathrm{v})$ EA/cyclohexane $] ;{ }^{1} \mathbf{H}$ NMR $\left(400 \mathrm{MHz}, \mathrm{CDCl}_{3}\right)$ $\delta 7.29(\mathrm{~s}, 1 \mathrm{H}), 5.65(\mathrm{q}, J=6.5 \mathrm{~Hz}, 1 \mathrm{H}), 3.76(\mathrm{~s}, 3 \mathrm{H}), 2.81-$ $2.57(\mathrm{~m}, 2 \mathrm{H}), 2.54(\mathrm{ddd}, J=12.1,9.2,6.4 \mathrm{~Hz}, 2 \mathrm{H}), 1.46(\mathrm{~d}, J=$ $6.5 \mathrm{~Hz}, 3 \mathrm{H}) ;{ }^{13} \mathbf{C}$ NMR $\left(100 \mathrm{MHz}, \mathrm{CDCl}_{3}\right) \delta$ 199.1, 187.9, 165.0, 125.2(2C), 120.3, 113.7, 51.9, 34.1, 26.7, 21.0; IR (film, cm-1): 2989, 2953, 1693, 1633, 1546, 1344, 991, 856, 752; HRMS (ESI) Found $m / z$ 231.0637, calcd for $\left[\mathrm{M}+\mathrm{Na}^{+}\right]$ $\mathrm{C}_{11} \mathrm{H}_{12} \mathrm{NaO}_{4} 231.0628$.

Dimethyl-6-(4-fluorophenyl)-2-methyl-2H-pyran-3,5dicarboxylate (2s): The title compound was prepared according to procedure $\mathrm{B}, 251 \mathrm{mg}, 82 \%$ yield, yellow oil, $\mathrm{R}_{\mathrm{f}}=0.2$ [1:5 (v/v) EA/cyclohexane]; ${ }^{1} \mathbf{H}$ NMR (400 $\left.\mathrm{MHz} \mathrm{CDCl}_{3}\right) \delta$ $7.64(\mathrm{~s}, 1 \mathrm{H}), 7.52-7.42(\mathrm{~m}, 2 \mathrm{H}), 7.12-7.03(\mathrm{~m}, 2 \mathrm{H}), 5.54$ (q, $J=6.5 \mathrm{~Hz}, 1 \mathrm{H}), 3.80(\mathrm{~s}, 3 \mathrm{H}), 3.64(\mathrm{~s}, 3 \mathrm{H}), 1.47(\mathrm{~d}, J=6.5 \mathrm{~Hz}$, $3 \mathrm{H}) ;{ }^{13} \mathrm{C}$ NMR $\left(100 \mathrm{MHz}, \mathrm{CDCl}_{3}\right) \delta 165.9,165.6,164.8$, $131.9,131.8,131.4(2 \mathrm{C}), 118.7,115.1(2 \mathrm{C}), 114.9,104.7,72.8$, 51.8, 51.5, 18.5; IR (film, cm-1): 2976, 1699, 1637, 1602, 1595, 1367, 1083, 1049, 1024, 952, 914, 840, 754; HRMS (ESI) Found $m / z$ 329.0807, calcd for $\left[\mathrm{M}+\mathrm{Na}^{+}\right] \mathrm{C}_{16} \mathrm{H}_{15} \mathrm{FNaO}_{5}$ 329.0796.

Methyl-2,6-dimethyl-5-(phenylsulfonyl)-2H-pyran-3carboxylate (2t): The title compound was prepared according to procedure $\mathrm{B}, 300 \mathrm{mg}$, $97 \%$ yield, colorless oil, $\mathrm{R}_{\mathrm{f}}=0.2[1: 5$ (v/v) EA/cyclohexane]; ${ }^{1} \mathbf{H}$ NMR $\left(400 \mathrm{MHz}, \mathrm{CDCl}_{3}\right) \delta 7.90-$ $7.79(\mathrm{~m}, 2 \mathrm{H}), 7.63-7.48(\mathrm{~m}, 3 \mathrm{H}), 7.41(\mathrm{~s}, 1 \mathrm{H}), 5.34(\mathrm{q}, J=$ $6.5 \mathrm{~Hz}, 1 \mathrm{H}), 3.75$ (s, 3H), 2.29 (s, 3H), $1.28(\mathrm{~d}, J=6.5 \mathrm{~Hz}, 3 \mathrm{H})$; ${ }^{13}$ C NMR $\left(100 \mathrm{MHz}, \mathrm{CDCl}_{3}\right) \delta 168.1,164.4,142.5,133.0$ (2C), 129.3, 128.3(2C), 126.5, 118.4, 114.4, 73.1, 51.9, 19.5, 19.5; IR (film, cm ${ }^{-1}$ ): 2980, 1703, 1633, 1556, 1435, 1274, 999, 927, 756; HRMS (ESI) Found $\mathrm{m} / \mathrm{z}$ 331.0600, calcd for $\left[\mathrm{M}+\mathrm{Na}^{+}\right] \mathrm{C}_{15} \mathrm{H}_{16} \mathrm{NaO}_{5} \mathrm{~S} 331.0611$.

Dimethyl 6-cyclopropyl-2-(2-ethoxy-2-oxoethyl)-2H-pyran3,5-dicarboxylate (3d): The title compound was prepared according to procedure C, $220 \mathrm{mg} ; 68 \%$ yield; colorless oil; $\mathrm{R}_{\mathrm{f}}$ $=0.2[1: 5(\mathrm{v} / \mathrm{v})$ EA/cyclohexane $] ;{ }^{1} \mathbf{H}$ NMR $\left(400 \mathrm{MHz}, \mathrm{CDCl}_{3}\right)$ $\delta 7.61(\mathrm{~s}, 1 \mathrm{H}), 5.67(\mathrm{dd}, J=10.2,3.0 \mathrm{~Hz}, 1 \mathrm{H}), 4.27-4.09(\mathrm{~m}$, 2H), 3.79 (s, 3H), $3.77(\mathrm{~s}, 3 \mathrm{H}), 3.12$ (tt, $J=8.2,4.9 \mathrm{~Hz}, 1 \mathrm{H})$, $2.74(\mathrm{dd}, J=15.4,10.2 \mathrm{~Hz}, 1 \mathrm{H}), 2.43(\mathrm{dd}, J=15.4,3.0 \mathrm{~Hz}$, $1 \mathrm{H}), 1.34-1.31(\mathrm{~m}, 1 \mathrm{H}), 1.28(\mathrm{t}, J=7.1 \mathrm{~Hz}, 3 \mathrm{H}), 1.05-1.00$ $(\mathrm{m}, 1 \mathrm{H}), 0.95-0.80(\mathrm{~m}, 2 \mathrm{H}) ;{ }^{13} \mathbf{C}$ NMR $\left(100 \mathrm{MHz}, \mathrm{CDCl}_{3}\right) \delta$ $173.9,169.4,165.8,164.7,132.8,113.7,103.8,72.2,60.9$, 51.7, 51.47, 37.6, 14.1, 13.2, 10.8, 7.7. IR (film, $\mathrm{cm}^{-1}$ ): 2968, 2954, 1732, 1707, 1693, 1547, 1449, 1393, 1175, 1114, 985, 
908, 877, 752; HRMS (ESI) Found $m / z 347.1107$ calcd for $\left[\mathrm{M}+\mathrm{Na}^{+}\right] \mathrm{C}_{16} \mathrm{H}_{20} \mathrm{NaO}_{7} 347.1101$.

Methyl-2-(2-ethoxy-2-oxoethyl)-5-oxo-5,6,7,8-tetrahydro2H-chromene-3-carboxylate (3i): The title compound was prepared according to procedure C, $211 \mathrm{mg} ; 72 \%$ yield; colorless oil; $\mathrm{R}_{\mathrm{f}}=0.2[1: 4(\mathrm{v} / \mathrm{v})$ EA/cyclohexane $] ;{ }^{1} \mathbf{H}$ NMR (400 $\left.\mathrm{MHz}, \mathrm{CDCl}_{3}\right) \delta 7.63(\mathrm{~s}, 1 \mathrm{H}), 5.79(\mathrm{dd}, J=9.8,3.2 \mathrm{~Hz}, 1 \mathrm{H})$, $4.20(\mathrm{q}, J=7.1 \mathrm{~Hz}, 2 \mathrm{H}), 3.79$ (s, 3H), 2.78 (dd, $J=15.0,9.8$ $\mathrm{Hz}, 1 \mathrm{H}), 2.61(\mathrm{dd}, J=15.0,3.2 \mathrm{~Hz}, 1 \mathrm{H}), 2.55-2.37(\mathrm{~m}, 4 \mathrm{H})$, $2.10-1.95(\mathrm{~m}, 2 \mathrm{H}), 1.29(\mathrm{t}, J=7.1 \mathrm{~Hz}, 3 \mathrm{H}) ;{ }^{13} \mathbf{C}$ NMR $(100$ $\left.\mathrm{MHz}, \mathrm{CDCl}_{3}\right) \delta 194.0,174.8,169.2,164.7,128.1,117.1,111.4$, 73.4, 61.0, 51.9, 39.3, 36.4, 28.4, 20.2, 14.2; IR (film, cm ${ }^{-1}$ ): 2983, 2871, 1726, 1709, 1633, 1592, 1445, 1383, 1271, 1229, 759, 726; HRMS (ESI) Found $\mathrm{m} / \mathrm{z}$ 317.1005, calcd for $\left[\mathrm{M}+\mathrm{Na}^{+}\right] \mathrm{C}_{15} \mathrm{H}_{18} \mathrm{NaO}_{6}: 317.0996$.

Methyl-2-(2-ethoxy-2-oxoethyl)-6-methyl-5(phenylsulfonyl)-2H-pyran-3-carboxylate $\mathbf{( 3 \mathbf { j } ) :}$ The title compound was prepared according to procedure C, $193 \mathrm{mg}$; $52 \%$ yield; yellow oil; $\mathrm{R}_{\mathrm{f}}=0.2[1: 5(\mathrm{v} / \mathrm{v}) \mathrm{EA} /$ cyclohexane $]$; ${ }^{1} \mathbf{H}$ NMR $\left(400 \mathrm{MHz}, \mathrm{CDCl}_{3}\right) \delta 7.95-7.81(\mathrm{~m}, 2 \mathrm{H}), 7.68-$ $7.52(\mathrm{~m}, 3 \mathrm{H}), 7.50(\mathrm{~d}, J=0.5 \mathrm{~Hz}, 1 \mathrm{H}), 5.72(\mathrm{dd}, J=9.9,3.3$ $\mathrm{Hz}, 1 \mathrm{H}), 4.15$ (q, $J=7.1 \mathrm{~Hz}, 2 \mathrm{H}), 3.80(\mathrm{~s}, 3 \mathrm{H}), 2.73(\mathrm{dd}, J=$ $15.0,9.9 \mathrm{~Hz} 1 \mathrm{H}), 2.50(\mathrm{dd}, J=15.0,3.3 \mathrm{~Hz}, 1 \mathrm{H}), 2.33(\mathrm{~s}, 3 \mathrm{H})$, $1.28(\mathrm{t}, J=7.1 \mathrm{~Hz}, 3 \mathrm{H}) ;{ }^{13} \mathbf{C}$ NMR $\left(100 \mathrm{MHz}, \mathrm{CDCl}_{3}\right) \delta 168.9$, $168.0,164.0,142.2,133.2,129.6(2 \mathrm{C}), 129.4(2 \mathrm{C}), 126.7$, 116.3, 115.2, 73.1, 61.0, 52.1, 38.5, 19.2, 14.2.; IR (film, $\left.\mathrm{cm}^{-1}\right)$ : $3061,2847,2360,2341,1663,1645,1620,1459,1386,1276$, $1150,883,759$; HRMS (ESI) Found $m / z$ 403.0823, calcd for $\left[\mathrm{M}+\mathrm{Na}^{+}\right] \mathrm{C}_{18} \mathrm{H}_{20} \mathrm{NaO}_{7} \mathrm{~S} 403.0822$.

\section{Supporting Information}

Supporting Information with full analysis of products $\mathbf{1 , 2}$ and $\mathbf{3}$ is available free of charge on the ACS Publications website.

\section{Corresponding Authors}

serge.thorimbert@upmc.fr; http://www.ipcm.fr/presentation-682?lang=en.

\section{ACKNOWLEDGMENT}

We thank University P. et M. Curie (UPMC) and CNRS for fundings. The Fédération de Recherche (FR2769) provided technical access for analysis. Acknowledgements to the French Embassy in Slovakia and to China Scholarship Council (CSC) for financial supports to K.P. and L.C. respectively.

\section{REFERENCES}

(1) Xie P.; Yang, J.; Zheng, J.; Huang, Y. Eur. J. Org. Chem. 2014, 1189-1194 and references cited herein.

(2) (a) For a review see: Hsung, R.P.; Kurdyumov, A.V.; Sydorenko, N. Eur. J. Org. Chem. 2005, 23-44. For selected recent examples see : (b) Luo, G.Y.; Wu, H.; Tang, Y.; Li, H.; Yeom, H.S.; Yang, K.; Hsung, R.P. Synthesis, 2015, 47, 2713-2720. (c) Li, K.; Ou, J.; Gao,
S. Angew. Chem. Int. Ed. 2016, 55, 14778-14783. (d) Suzuki, T.; Watanabe, S.; Kobayashi, S.; Tanino, K. Org. Lett. 2017, 19, 922925. (e) Takeda, Y.; Narita, K.; Katoh, T. Eur. J. Org. Chem. 2017, 901-907..

(3) (a) Gosink, T.A. J. Org. Chem. 1974, 39, 1942-1944. (b) Rodriguez-Otero, J. J. Org. Chem. 1999, 64, 6842-6848. (c) Krasnaya, Zh.A. Chemistry of Heterocyclic Compounds 1999, 35, 1255-1271. (d) Riveira, M.J.; Quiroga, G.N.; Mata, E.G.; Gandon, V.; Mischne, M.P. J. Org. Chem. 2015, 80, 6515-6519.

(4) For selected examples see: (a) Peng, W.; Hirabaru, T.; Kawafuchi, H.; Inokuchi, T. Eur. J. Org. Chem. 2011, 5469-5474. . (b) Fotiadou, A.D.; Zografos, A.L. Org. Lett. 2012, 14, 5464-5667. (c) Riveira, M.J.; Mischne, M.P. Chem. Eur. J. 2012, 18, 2382-2388. (d) Edayadulla, N.; Lee, Y.R. Bull. Korean Chem. Soc, 2013, 34, 29632967. (e) Narayana, V.R.; Pudukulathan, Z.; Varala, R. Org. Commun. 2013, 6, 110-119.

(5) For other activated methylene groups involved in formal [3+3]CA see for hydroxyquinolones: (a) Riveira, M.J.; Mischne, M.P. Synth. Commun. 2013, 43, 208-220. (b) For $\beta$-oxosulfones: Pena, J.; Moro, R.F.; Basabe, P.; Marcos, I.S.; Diez, D. RSC Advances, 2012, 2, 8041-8049. (c) From dienyl-diketones: Jacob, S.D.; Brooks, J.L.; Frontier, A. J. J. Org. Chem. 2014, 79, 10296-10302.

(6) (a) Menz, H.; Kirsch, S.F. Org. Lett. 2006, 8, 4795-4797. (b) Zhu, Z.B.; Kirsch S.F. Chem. Commun. 2013, 49, 2272-2283. (c) Vidhani, D.V.; Krafft, M.E.; Alabugin, I.V. Org. Lett. 2013, 15, 44624465. (d) Vidhani, D.V.; Krafft, M.E.; Alabugin, I.V. J. Org. Chem. 2014, 79, 352-364. (e) Chong, Q.; Wang, C.; Wang, D.; Wang, H.; Wu, F.; Xin, X.; Wan, B. Tetrahedron Lett. 2015, 56, 401-403.

(7) Rauser, M.; Schroeder, S.; Niggeman, M. Chem. Eur. J. 2015, 21, 15929-15933.

(8) Sun, J.; Zhu, D.; Gong, H.; Yan, C.G. Tetrahedron 2013, 69, 10565-10572.

(9) (a) Hu, J.; Dong, W.; Wu, X.Y.; Tong, X. Org. Lett. 2012, 14, 5530-5533. (b) Ni, C.; Tong, X. J. Am. Chem. Soc. 2016, 138, 78727875 .

(10) (a) Agarwal, J.; Bayounes, O.; Thorimbert, S.; Dechoux, L. RSC Advances, 2014, 4, 2772-2775. (b) Plevovà, K.; Chang, L.; Martin, E.; Llopis, Q.; Dechoux, L.; Thorimbert, S. Adv. Synth. Catal. 2016, 358, 3293-3297.

(11) Addition of aceto-phenylsulfone to methyl coumalate, using DBU in DCM, was described giving one $2 H$-pyran structure in $67 \%$. See : Frisbee, A.R.; Nantz, M.H.; Kramer, G.W. Fuchs, P.L. J. Am. Chem. Soc. 1984, 106, 7143-7145.

(12) Neises, B.; Steglich, W. Angew. Chem. Int. Ed. 1978, 17, $522-524$

(13) This hypothesis is supported by two results: a first experiment using stoichiometric amount of $\mathrm{NaH}$ as base in DCM with 2,4pentanedione gave $\mathbf{4 g}$ in the enol form after acidic quench. When the reaction was followed by ${ }^{1} \mathrm{H} \mathrm{NMR}$, this compound was detected as an intermediate.

(14) Alabugin, I.V.; Manoharan, M.; Breiner, B.; Lewis, F.D. J. Am. Chem. Soc. 2003, 125, 9329-9342.

(15) (a) Wu, S.; Zhang, Y.; Agarwal, J.; Mathieu, E.; Thorimbert, S.; L. Dechoux. L. Tetrahedron, 2015, 71, 7663-7669. (b) Wu, S.; Yang, L.; Sun, W., Si, W.; Xiao, S.; Wang, Q.; Dechoux. L; Thorimbert, S.; Sollogoub, M.; Zhou, D.; Zhang, Y. Eur. J. Med. Chem. 2017, 130, 308-319. 